\title{
Trends in Macrolide Resistance for Streptococcus pyogenes, Streptococcus agalactiae and Streptococcus pneumoniae and its Association with Social Clustering in Argentina
}

\author{
Gabriela Rubinstein ${ }^{1,2, *}$, Bárbara Bavdaz ${ }^{3}$, Sabrina De Bunder ${ }^{4}$ and Néstor Blazquez ${ }^{4}$
}

${ }^{1}$ Laboratorio Hospital Privado Regional, 20 de Febrero 591, 8400 Bariloche, Argentina; ${ }^{2}$ Laboratorio del Sol,
Sanatorio del Sol, 20 de Febrero 640, 8400 Bariloche, Argentina; ${ }^{3}$ Laboratorio San Carlos, Sanatorio San Carlos, Av.
Bustillo Km. 1, 8400 Bariloche, Argentina; ${ }^{4}$ Laboratorio de Bacteriología, Hospital Ramón Carrillo F.P. Moreno 651,
8400 Bariloche, Argentina

\begin{abstract}
The aim of this work was to study and compare macrolide resistance patterns among Streptococcus pneumoniae, Streptococcus agalactiae and Streptococcus pyogenes, to describe the phenotypes of macrolide-lincosamide resistance and to further investigate associations between macrolide resistance and social clustering. Susceptibility data were obtained from 4 clinical microbiology laboratories in Bariloche, Argentina for the period 2002- 2008. Patients were differentiated in two population clusters according to the institution they attended (public or private). A total of 4310 strains were studied: 2615 S. pyogenes, 995 S. agalactiae and 700 isolates of S.pneumoniae. Erythromycin resistance rates over the study period were $1.5 \%$ for S. pyogenes, $7.8 \%$ for S. agalactiae and $11.3 \%$ for S. pneumoniae. For the complete study group, these values differed significantly among species (contingency table $\chi^{2}=164.52, \mathrm{p}<0.0001$ ). Resistance in S.pyogenes and S. pneumoniae showed irregular trends whilst S. agalactiae showed an increasing tendency during the whole period appearing to be a better indicator of the trends in macrolide resistance. A dual character MLS $_{\mathrm{B}}$ phenotype of both inducible and constitutive resistance was observed in $S$. agalactiae only. Resistance in isolates from the population attending private institutions was significantly higher than those attending the public hospital ( $S$. pyogenes, (1.9 vs. $\left.0.6, \chi^{2}=5.851, \mathrm{p}<0.025\right)$; S. pneumoniae, 19.1 vs. $7.0\left(\chi^{2}=21.98, \mathrm{p}<0.001\right)$, and. S. agalactiae, 12.4 vs. 3.8 $\left.\left(\chi^{2}=24.5, \mathrm{p}<0.001\right)\right)$. In conclusion, macrolides resistance rates during 2002-2008 were significantly different for S. pyogenes, S. agalactiae and S.pneumoniae. We also found significant variations in macrolide resistance levels in different population groups.
\end{abstract}

Keywords: Erythromycin, streptococci, resistance- trends.

\section{INTRODUCTION}

Increasing antimicrobial resistance is becoming a serious international problem in both hospital and community settings $[1,2]$. Many factors are probably involved in the emergence and spread of antibiotic resistance, but antibiotic consumption seems to be the main driving force [3-5].

Macrolides are frequently used for the treatment of community-acquired respiratory tract infections and are the first alternative choice for those patients that are hypersensitive to penicillin. Since the introduction of the newer, long-acting macrolides, resistance to these drugs in streptococci has increased worldwide. In addition, resistance rates vary in different geographic areas both among countries and among regions within countries suggesting that resistance surveillance must be undertaken both nationally and locally [3, 6-11].

Macrolide resistance in Streptococci is mediated by two major mechanisms: methylation of the ribosomal macrolide

*Address correspondence to this author at the Laboratorio Hospital Privado Regional, 20 de Febrero 591, 8400 Bariloche, Argentina; Tel: +54 2944 448761; Fax: +54 2944 437418; E-mail: gabrielar@bariloche.com.ar target site which results in resistance to 14-, 15- and 16membered macrolide, lincosamides and streptogramin B $\left(\mathrm{MLS}_{\mathrm{B}}\right.$ phenotype), and drug efflux which results in resistance to 14 - and 15 -membered macrolide resistance only (M phenotype) [12]. The prevalence of these two major mechanisms among macrolide resistant streptococci varies in different species and geographically.

In Argentina, although prevalence of erythromycin resistance in streptococci is still low, it has increased significantly in recent years in most regions [9, 13-15]. In previous work carried out in Bariloche we found low overall resistance rates for $S$. pyogenes, but a significant rise in resistance in one particular population group [9]. In that report, we found that isolates originated from groups attending public hospital, showed very little resistance while there was an increase in resistance in isolates from a more affluent group attending private hospitals. Association between antibiotic resistance and social grouping has been previously described in Wales although their results showed higher resistance values associated with deprivation [16].

The aim of this study was to describe erythromycin resistance patterns among different species of streptococci, S. pneumoniae, S. agalactiae and S. pyogenes, in Bariloche, 
Table 1. Erythromycin and Clindamycin Resistance in S. pyogenes, S. pneumoniae and S. agalactiae for the Period January 2002 to December 2008

\begin{tabular}{|c|c|c|c|c|c|c|}
\hline \multirow{2}{*}{} & \multicolumn{2}{|c|}{ S. pyogenes } & \multicolumn{2}{c|}{ S. pneumoniae } & \multicolumn{2}{c|}{ S. agalactiae } \\
\cline { 2 - 7 } & Public & Private & Public & Private & Public & Private \\
\hline \hline Erythromycin Resistance (\%) & 0.6 & 1.9 & 7.0 & 19.1 & 3.8 & 12.4 \\
\hline Clindamycin Resistance (\%) & 0.2 & 0.5 & 2.6 & 8.3 & 3.0 & 11.1 \\
\hline Total tested & 820 & 1795 & 454 & 246 & 529 & 466 \\
\hline
\end{tabular}

Argentina during the period 2002-2008. We describe the phenotypes of macrolide-lincosamide resistance and further investigate associations between macrolides resistance and social clustering.

\section{Materials and Methodology}

Susceptibility data for $S$. pyogenes, S. agalactiae and $S$. pneumoniae were obtained from clinical samples routinely collected in Bariloche, a city located in Northern Patagonia, Argentina, between January 2002 and December 2008. Data were available from 4 clinical microbiology laboratories, three private and one public, which together serve more than $80 \%$ of the city population.

Strains were identified using standard manual procedures. Macrolides susceptibility was tested by the disk diffusion method on Mueller-Hinton agar supplemented with 5\% defibrinated sheep blood (Laboratorio Argentino, Buenos Aires, Argentina), using $15 \mu \mathrm{g}$ erythromycin disks and $2 \mu \mathrm{g}$ clindamycin disks (Laboratorios Britania, Buenos Aires, Argentina) according to CLSI guidelines. The phenotypes of erythromycin resistant strains were determined by the double-disk test with erythromycin and clindamycin disks separated by $15-20 \mathrm{~mm}$ [12].

Two different patient groups were observed according to the institutions they attended. The patients that attend the public hospital mostly lack health insurance and commonly have low incomes. The other group corresponds to patients with medical insurance which can be assigned to a more affluent population.

Differences in the prevalence of resistance between different groups were assessed by the $\chi^{2}$ test.

\section{RESULTS}

During the study period 4310 strains of three different species of Streptococci were collected and analyzed. The city's public hospital provided 1803 strains whilst 2507 originated from private hospitals.

A total of $2615 S$. pyogenes isolates were obtained. The majority $(96.8 \%)$ were isolated from throat swabs, $1.3 \%$ from soft tissue samples and the remaining $1.9 \%$ from other samples. The mean age of the patients from which these isolates were obtained was 12.8 years (range: 0 to 78). Nine hundred and ninety five isolates of $S$. agalactiae were obtained from the following samples: rectal-vaginal swabs (46.8\%), vaginal swabs $(30.7 \%)$, urine (15.2\%), blood $(1.6 \%)$ and other sites $(5.7 \%)$. For this species the patients mean age was 30.5 years (range: 8 to 89 ). Finally, 700 isolates of $S$. pneumoniae were obtained from: sputum $(59.2 \%)$, bronco-alveolar lavage $(3.9 \%)$, pleural fluid $(0.9 \%)$, blood $(24 \%)$, CSF $(0.6 \%)$ and other sites $(11.4 \%)$. The mean age for these patients was 47.65 (range: 0 to 92 ).

The global rates of resistance to erythromycin over the complete study period were $1.5 \%$ for $S$. pyogenes, $7.8 \%$ for $S$. agalactiae and $11.3 \%$ for $S$. pneumoniae. For the complete study group, resistance differed significantly among species (contingency table $\chi^{2}=164.52$, $\mathrm{p}<0.0001$ ). These differences were also observed when analysing samples obtained from public $\left(\chi^{2}=44.19, \mathrm{p}<0.0001\right)$ and private institutions $\left(\chi^{2}=188.31, \mathrm{p}<0.0001\right)$ separately.

The resistant rates for erythromycin and clindamycin, for the different species and patients groups are described in Table 1.

Annual resistance rates for $S$. pyogenes showed a significant increase in 2002 compared with data from previous studies $(0.6 \%$ in 2000 , and $0.0 \%$ in 2001$)$ with a peak in 2003 and a sharp decline in 2004 (Fig. 1) [9]. The resistant isolates of this peak (2003) originated mostly from private laboratories $(90.5 \%)$ and belonged mainly $(85.7 \%)$ to the $\mathrm{M}$ phenotype.

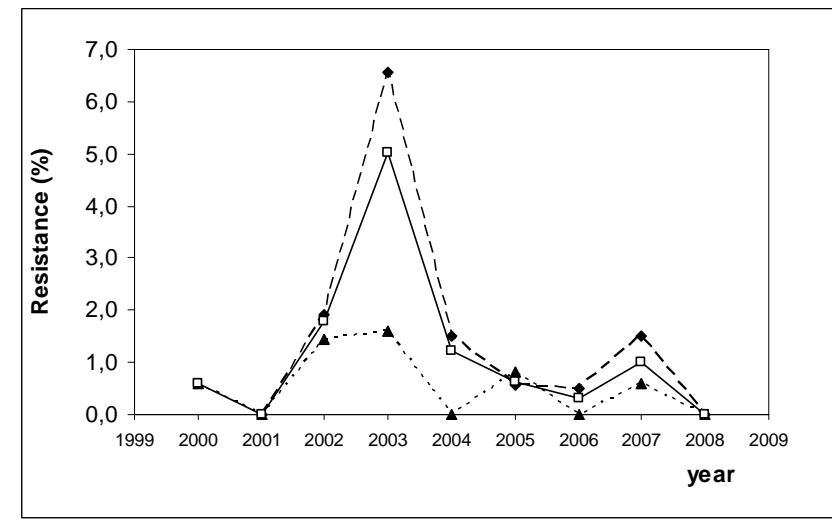

Fig. (1). Erythromycin resistance in S.pyogenes. Filled triangles correspond to values for public hospital, filled diamonds for private hospitals and open squares for the total population.

For S. pneumoniae erythromycin resistance rates increased from $6.0 \%$ in 2002 to $7.2 \%$ in 2008 but variations were observed during the study period. Erythromycin resistance rose abruptly from $9 \%$ in 2004 to $17.5 \%$ in 2005 and $20 \%$ in 2006 , and fell again to $8.5 \%$ in 2007 . In this case, the increased resistance values seen during this peak 
occurred both in private and public laboratories and $62.5 \%$ and $75 \%$ of the resistant isolates (respectively) showed the M phenotype (Fig. 2).

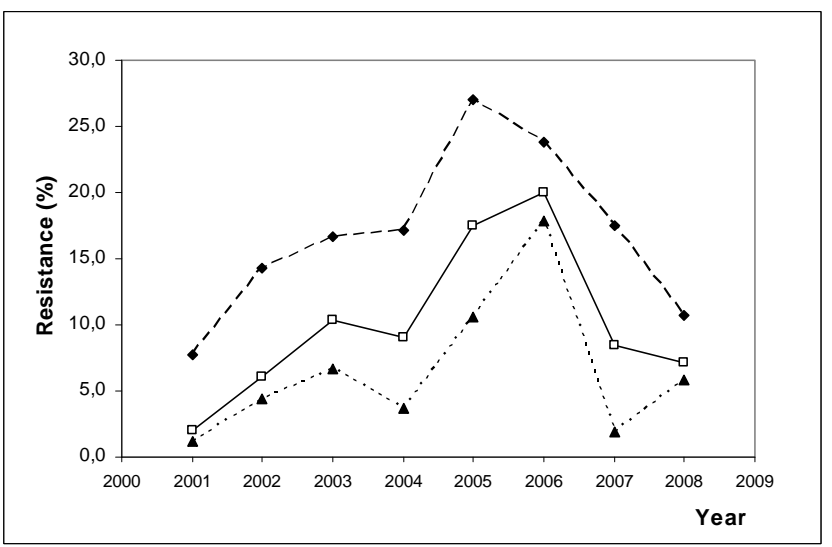

Fig. (2). Erythromycin resistance in S.pneumoniae. Filled triangles correspond to values for public hospital, filled diamonds for private hospitals and open squares for the total population.

Resistance for S. agalactiae rose from $1.2 \%$ in 2002 to $10,7 \%$ in 2008 and we noted a steady increasing tendency in resistance during the whole period $\left(R^{2}=0.77 ; p<0.009\right)$ with a rapid increase only at the beginning of the study period . This pattern was observed both in strains isolated at private and public institutions ( $0 \%$ in 2002 to $13.2 \%$ in 2008, and $2.0 \%$ in 2002 to $7.9 \%$ en 2008 respectively) (Fig. 3).

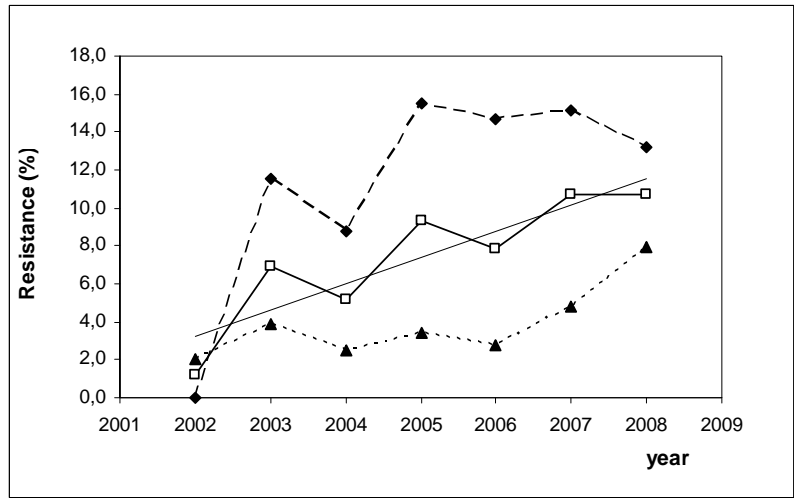

Fig. (3). Erythromycin resistance in agalactiae. Filled triangles correspond to values for public hospital, filled diamonds for private hospitals and open squares for the total population. Full line indicates a linear regression line corresponding to data for the total population $\left(\mathrm{r}^{2}=0.77 ; \mathrm{p}<0.009\right)$.

During the study period, the incidence of macrolides resistance in isolates from private hospitals was higher than those obtained in isolates from public hospital. These differences were significant for the three species, $S$. pyogenes, $\left(\chi^{2}=5.851, \mathrm{p}<0.025\right)$, S. pneumoniae, $\left(\chi^{2}=21.98\right.$, $\mathrm{p}<0.001)$, and. S. agalactiae, $\left(\chi^{2}=24.5, \mathrm{p}<0.001\right)$ (Fig. 4).

Four different phenotypes were identified by the doubledisk test. Resistance to erythromycin with blunting of the clindamycin zone of inhibition on the side of the erythromycin disk indicated an inducible MLS $_{B}$ phenotype (i $\mathrm{MLS}_{\mathrm{B}}$ ), resistance to both erythromycin and clindamycin indicated a constitutive $\operatorname{MLS}_{\mathrm{B}}$ phenotype $\left(\mathrm{c} M L S_{\mathrm{B}}\right)$, and resistance to erythromycin and susceptibility to clindamycin with no blunting, indicated an $M$ phenotype. A dual character $\mathrm{MLS}_{\mathrm{B}}$ phenotype of both inducible and constitutive resistance was observed in $S$. agalactiae only. It showed resistance to erythromycin with blunting of the clindamycin zone of inhibition on the side of the erythromycin, such as in the $\mathrm{i}_{\mathrm{MLS}}$, but slight bacterial growth inside the D shaped clindamycin inhibition zone is observed. This phenotype has been recently described in Staphylococcus aureus and denominated $\mathrm{fMLS}_{\mathrm{B}}$ [17]. The phenotype distribution for each species can be seen in Fig. (5). The M phenotype was the predominant resistance phenotype in S.pyogenes $(76.3 \%)$ and S. pneumoniae $(60.8 \%)$ but less frequent in S.agalactiae $(12.8 \%)$. The $\mathrm{cMLS}_{\mathrm{B}}$ phenothype was second in frequency for the three species: S.pyogenes $(13.2 \%)$, S. pneumoniae $(31.6 \%)$ and S.agalactiae $(30.8 \%)$. The inducible MLS $_{\mathrm{B}}$ phenotype $\left(\mathrm{i} \mathrm{MLS}_{\mathrm{B}}\right.$ ) was the predominant phenotype in S.agalactiae, and represented only $10.5 \%$ and $7.6 \%$ of $S$. pyogenes of $S$. pneumoniae resistant isolates respectively. Finally, the $\mathrm{f} \mathrm{MLS}_{\mathrm{B}}$ phenotype was only observed in S.agalactiae $(17.9 \%)$.

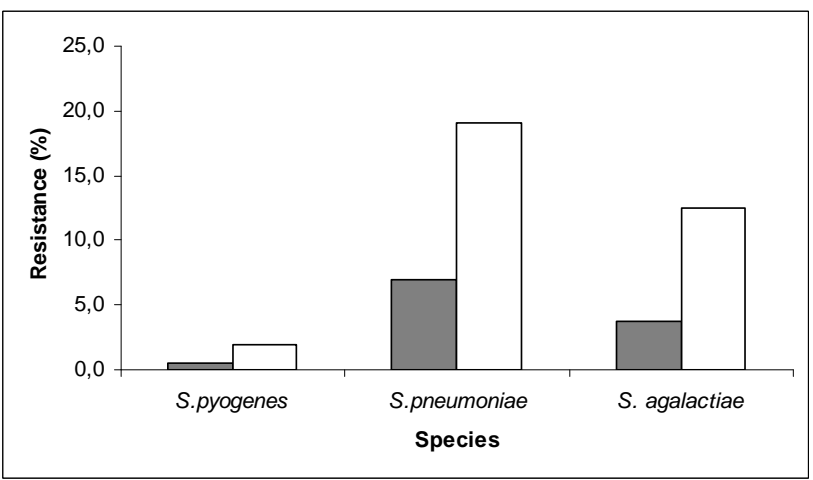

Fig. (4). Erythromycin resistance in S. pyogenes, S. agalactiae and S.pneumoniae for public hospital (grey bars) and private hospitals (white bars) during the period 2002-2008.

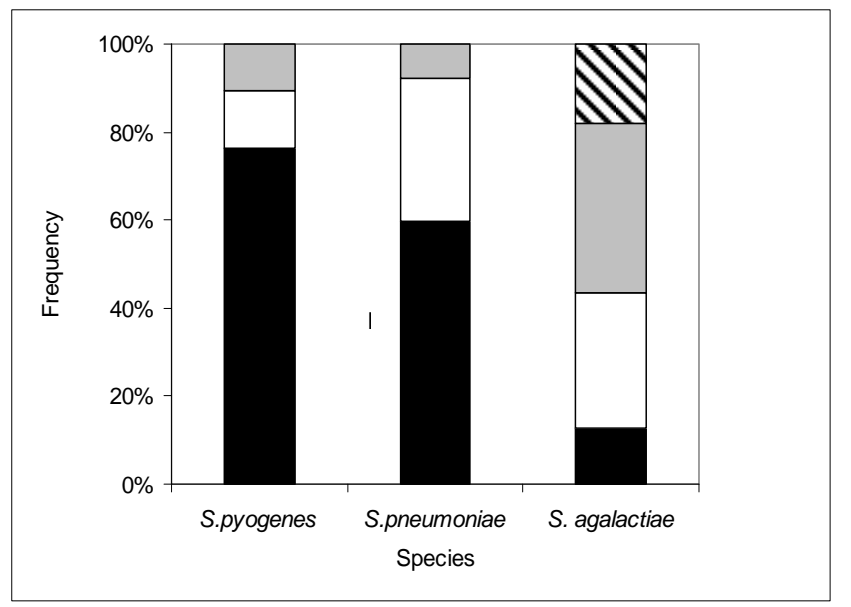

Fig. (5). Erythromycin resistance phenotype distribution in $S$. pyogenes, S. pneumoniae and S. agalactiae during the period 20022008. Full bars correspond to M phenotype, white bars correspond to $\mathrm{c} M L \mathrm{~S}_{\mathrm{B}}$ phenotype, grey bars correspond to i $\mathrm{MLS}_{\mathrm{B}}$ phenotype and hatched bars correspond to $\mathrm{MLS}_{\mathrm{B}}$ phenotype. 


\section{DISCUSSION}

The prevalence of erythromycin resistance in $S$. pyogenes, $S$. agalactiae and $S$. pneumoniae for the whole study period was low. However, resistance values were significantly different for the three species with higher values for $S$. pneumoniae. The observed differences in resistance among species may be explained by different factors such as patient age and gender, sites of isolation or even co-selection of resistance in multirresistant $S$. pneumoniae. A detailed analysis of these factors was not considered here and deserves specific designed studies.

The distribution of resistance phenotypes was also different for these three species, with a higher prevalence of the $\mathrm{M}$ phenotype in $S$. pyogenes and of the different expressions of the $\mathrm{MLS}_{\mathrm{B}}$ phenotype in S. agalactiae. On the other hand, $S$. pneumoniae showed a similar distribution of the two main resistance mechanisms. We observed a different $\mathrm{f}_{\mathrm{MLS}}$ B phenotype for $S$. agalactiae, similar to that first described in $S$. aureus [17] which is characterized by both constitutive and inducible expression of $\mathrm{MLS}_{\mathrm{B}}$ at once.

An interesting discrepancy in the resistance trends for these species was observed. S. agalactiae showed a clear increasing tendency during the whole period in the two study groups with no sharp changes. In addition, the distribution of the resistant phenotypes in this species was diverse. On the other hand, both $S$. pyogenes and $S$. pneumoniae showed irregular trends. Resistance in $S$. pyogenes was very low except during 2002 and 2003. The abrupt increase in resistance in these two years occurred mainly in one of the studied populations and resistant isolates possessed mostly the $\mathrm{M}$ phenotype. In both populations studied, resistance in $S$. pneumoniae showed a slow increasing tendency during the first years but increased sharply during 2005 and 2006, with abrupt decline in 2007. During these two years, prevalence of the $\mathrm{M}$ phenotype was higher than that obtained for the complete study period. Although clonality of resistant isolates was not examined here, the occurrence of these peaks for both $S$. pyogenes and $S$. pneumoniae are probably due to fluctuations in the occurrence of specific resistant clones.

The dissemination of dominant clones of community acquired pathogens such as $S$. pyogenes and $S$. pneumoniae has been frequently observed and probably explains the lack of a clear trend in resistance observed for these two species in the present study $[18,19]$. $S$. agalactiae isolates originate mostly from screening samples or from sites of endogenous infections where the spread of resistant clones is probably rare and has not been described. These isolates seem to be a more heterogeneous population and could probably be a better indicator of the tendency of macrolides resistance in our community.

In Bariloche, where antimicrobial restriction policies for macrolides are non-existent, the increasing resistance trend observed in $S$. agalactiae suggests that the reduction in resistance for $S$. pneumoniae and $S$. pyogenes may be the result of a natural decline of resistant clones and not a consequence of a diminishing selection pressure.

Because antibiotic resistance is an ongoing issue in the treatment of infectious diseases, much effort is devoted to the development of policies that restrict antimicrobial use in the community. In a recent article, Enne reviews the results of studies on resistance control in the community after a prescribing restriction policy [20]. In particular, in Finland, where good results were obtained for $S$. pyogenes when restricting macrolides usage, Enne considered the possibility that the observed reduction in resistance may have been due to the natural decline of the prevalent resistant clone rather than as a direct consequence of the antimicrobial restriction policies. Our results suggest that monitoring of macrolide resistance patterns after any intervention might be better accomplished by studying related species, such as $S$ agalactiae, where clonal dissemination appears to be less common.

A remarkable finding of our work is the observation of higher resistance values noted for isolates obtained from the population attending private hospitals of Bariloche for all three Streptococcus species studied. This could be linked to differences in macrolide consumption in the studied groups. Private hospitals are more frequently attended by a more affluent population that can afford buying antibiotics and therefore could conceivably consume greater amounts of macrolides, especially after the recent introduction of new types of macrolides with greater pharmacokinetic advantages. On the other hand, public hospitals often provide their patients with antibiotics but rarely offer macrolides (Head Pharmacist at Hospital Ramon Carrillo, Bariloche, pers.com.). Pharmacy records and/or antibiotic utilization could not be tallied in this study but may warrant future investigation. Our results are thus opposite to those obtained by Howard et al. (2001), who found deprivation associated with higher amoxicillin resistance values in Escherichia coli from urinary tract infection. ${ }^{16}$ Our findings suggest that social clustering might have diverse implications in relation to antibiotic consumption for different geographical areas while emphasizing how antibiotic consumption may relate to resistance in very complex ways.

In conclusion, resistance to macrolides in streptococci in Bariloche is still low, however significant differences between the studied species were observed. We also describe for the first time in Streptococcus agalactiae, a different phenotype, the $\mathrm{f}_{\mathrm{MLS}}$, similar to that previously described in $S$. aureus with both constitutive and inducible characteristics. $S$. agalactiae appears to be a better indicator of the trend of macrolide resistance in this community and should be considered when macrolides restriction policies are to be applied and their results evaluated. Finally, we also describe significant variations in macrolide resistance levels for diverse population groups of the same geographic area, which may be associated with differences between antibiotic consumption patterns in different social groups in Argentina. However the contribution of other factors to these temporal patterns is difficult to assess and therefore requires further studies.

\section{ACKNOWLEDGMENTS}

We thank Dr Rocco Lassalla, Dr Margaret Heginbothom and Dr Horacio Lopardo for their helpful comments on the manuscript. Some of the ideas developed throughout this paper originated during a visit to the UK sponsored by BSAC Overseas Fellowship Grant to Gabriela Rubinstein. 


\section{REFERENCES}

[1] Wise R, Cars O, Streulens M, Helmuth M, Huovinen P, Sprenger M. Antimicrobial resistance. BMJ 1998; 317: 609-10.

[2] Goossens H, Ferech M, Vander Stichele R, Elseviers M, EASC Project Group. Outpatient antibiotic use in Europe and association with resistance: a cross-national database study. Lancet 2005; 365 : 579-87.

[3] Garcia-Rey C, Fenoll A, Aguilar L, Casal J. Effect of social and climatological factors on antimicrobial use and Streptococcus pneumoniae resistance in different provinces in Spain. $\mathrm{J}$ Antimicrob Chemother 2004; 54: 465-71.

[4] Granizo JJ, Aguilar L, Casal J, García-Rey C, Dal-Re R, Baquero F. Streptococcus pneumoniae resistance to erythromycin and penicillin in relation to macrolide and $\beta$-lactam consumption in Spain (1979-1997). J Antimicrob Chemother 2000; 46: 767-73.

[5] Granizo JJ, Aguilar L, Casal J, Dal-Re R, Baquero F. Streptococcus pyogenes resistance to erythromycin in relation to macrolide consumption in Spain (1986-1997). J Antimicrob Chemother 2000; 46: 959-64.

[6] Alos J I, Aracil B, Torres C, Gomez-Garces JL, The Spanish Group for the Study of Infection in Primary Health Care Setting. High prevalence of erythromycin-resistant, clindamycin/miocamycinsusceptible (M phenotype) Streptococcus pyogenes: results of a Spanish multicentre study in 1998. J Antimicrob Chemother 2000; 45: 605-9.

[7] Yan J, Wu H, Whang A, Fu H, Lee C, Wu, J. Prevalence of polyclonal mef-A containing isolates among erythromycin resistant group A Streptococci in Southern Taiwan. J Clin Microbiol 2000; 38: 2475-9.

[8] Palavecino EL, Riedel I, Berrios X, et al. Prevalence and mechanisms of macrolide resistance in Streptococcus pyogenes in Santiago, Chile. Antimicrob Agents Chemother 2001; 45: 339-41.

[9] Rubinstein G, Amoroso A, Bavdaz B, De Bunder S, Blázquez N, Gutkind GO. Low Macrolide Resistance in Streptococcus pyogenes in Southern Argentina. IJAC 2005; 25: 450-1.

[10] Bergman M, Huikko S, Pihlajamaki M, et al. Effect of Macrolide consumption on erythromycin resistance in Sytreptococcus pyogenes in Finland in 1997-2001. Clin infect Dis 2004; 38: 1251-6.

[11] McCormick A, Whitney CG, Farley MM, et al. Geographic diversity and temporal trends of antimicrobial resistance in Streptococcus pneumoniae in United States. Nature Medicine 2003; 9: 424-30.

[12] Seppala H, Nissinen A, Yu Q, Huovinen P. Three different phenotypes of erythromycin-resistant Streptococcus pyogenes in Finland. J Antimicrob Chemother 1993; 32: 885-91.

[13] Perez J, Limanski A, Toresani I, et al. Distribución de tipo capsular y sensibilidad Antimicrobiana de Streptococcus agalactiae productores de infecciones en Argentina. Revista Argentina de Microbiologia 2004; 36: 63-67.

[14] Lopardo H A, HernandezC, Vidal P et al. Eritromycin-Resistant Streptococcus pyogenes in Argentina. Medicina 2004; 64: 143-5.

[15] Agudelo CI, Castañeda E, Corso A, et al. Resistencia a antibióticos no betalactámicos de aislamientos invasores de Streptococcus pneumoniae en niños latinoamericanos. SIREVA II, 2000-2005. Pan Am J Public Health 2009; 25(4): 305-13.

[16] Howard AJ, Magee JT, Fitzgerald KA, Dunstan FDJ. Factors Associated with antibiotic resistance in coliform organisms from community urinary tract infection in Wales. $\mathbf{J}$ Antimicrob Chemother 2001; 47: 301-13.

[17] Yoon EJ, Kwon AR, Min YH, Choi EC. Foggy D-shaped zone of inhibition in Staphylococcus aureus owing to a dual character of both inducible and constitutive resistance to macrolide-lincosamidestreptogramin B. J Antimicrob Chemother 2008; 61(3): 533-40.

[18] Seppala H, Klaukka T, Vuopio-Varkila J, et al. The effects of changes in the consumption of macrolides antibiotics on erythromycin resistance in group A streptococci in Finland. N Engl J Med 1997; 337: 441-6.

[19] Soares S, Kristinsson KG, Musser JM, Tomasz A. Evidence for the introduction of a multiresistant clone of serotype 6B Streptococcus pneumoniae from Spain to Iceland in the late 1980s. J Infect Dis 1993; 168: 158-63.

[20] Enne VI. Reducing antimicrobial resistance in the community by restricting prescribing: can it be done? J Antimicrob Chemother 2010; 65: 179-82.

Received: June 24, 2010

Revised: December 29, 2010

Accepted: December 29, 2010

(C) Rubinstein et al.; Licensee Bentham Open.

This is an open access article licensed under the terms of the Creative Commons Attribution Non-Commercial License (http://creativecommons.org/licenses/ by-nc/3.0/) which permits unrestricted, non-commercial use, distribution and reproduction in any medium, provided the work is properly cited. 